\title{
Inhibition of DNA methyltransferases, histone deacetylases and lysine-specific demethylase-1 suppresses the tumorigenicity of the ovarian cancer ascites cell line SKOV3
}

\author{
FANLIANG MENG ${ }^{1,2}$, GUIQIN SUN ${ }^{1,2}$, MEI ZHONG $^{2}$, YANHONG YU $^{2}$ and MOLLY A. BREWER ${ }^{1}$ \\ ${ }^{1}$ Division of Gynecologic Oncology, University of Connecticut Health Center, Farmington, CT 06032, USA; \\ ${ }^{2}$ Department of Obstetrics and Gynecology, Nanfang Hospital, Southern Medical University, Guangzhou 510515, P.R. China
}

Received February 10, 2013; Accepted April 8, 2013

DOI: $10.3892 /$ ijo.2013.1960

\begin{abstract}
Ovarian cancer is one of the most lethal female malignancies and epigenetic abnormalities are thought to play a vital role in the pathogenesis, development and progression of ovarian cancer. Our goal was to investigate whether the combination of trichostatin A (TSA) and 5-aza-2'-deoxycytidine (decitabine) was superior to single agent on tumorigenicity of ovarian cancer cells. We found that tumorigenicity and metastasis of SKOV3 cells were significantly suppressed by the combination of TSA and decitabine in xenograft mouse models. Migration capacity was markedly suppressed through the induction of E-cadherin and suppression of $\mathrm{N}$-cadherin when treated with TSA and decitabine. Invasion was also suppressed at least partially through inhibition of MMP-2 and MMP-9 with the combined treatment. The combination drugs markedly inhibited spheroid formation and significantly impaired migration and invasion capacity of spheroid derived cells through inhibition of Twist, N-cadherin, MMP-2, MMP-9 and induction of E-cadherin. Epigenetically, the activity of DNA methyltransferases (DNMTs) and histone deacetylases (HDACs) were markedly inhibited when TSA was used in combination with decitabine, especially the expression of DNMT3A/3B and HDAC1/2. Acetylation of histone $\mathrm{H} 3$ and $\mathrm{H} 4$ were more markedly stimulated with the combination than with either agent alone. The expression level of lysine-specific demethylase-1 (LSD1) was also suppressed. The transcription activity marker dimethylated-H3K4 was induced, but
\end{abstract}

Correspondence to: Dr Molly A. Brewer, Division of Gynecologic Oncology, University of Connecticut Health Center, 263 Farmington Ave, Farmington, CT 06032, USA

E-mail: mbrewer@uchc.edu

Dr Yanhong Yu, Department of Obstetrics and Gynecology, Nanfang Hospital, Southern Medical University, 1838 North Guangzhou Ave, Guangzhou 510515, P.R. China

E-mail: yuyh1010@hotmail.com

Key words: migration, invasion, spheroid, DNA methyltransferase, histone deacetylase, histone methylation the dimethylated-H3K9 was suppressed by exposure to the combined drugs. These results suggest that the combination of TSA and decitabine significantly suppresses tumorigenicity by inhibiting migration and invasion of ovarian cancer cells via regulating the expression of the cadherins and MMPs, which may be epigenetically regulated by DNA methylation and histone modification.

\section{Introduction}

Metastasis occurs when tumor cells disseminate from primary tumor and invade distal organs by the circulation of ascities in ovarian cancer (1). The capacity to detach from adjacent cells in a primary location, degrade extracellular matrix (ECM) and invade omentum and peritoneum are necessary for cancer cells to be capable of metastasizing (2). Migration and invasion are two of the most important processes in the metastasis of cancer cells and the full understanding of these processes are essential to the prevention and eradication the metastasis of cancer.

E-cadherin and $\mathrm{N}$-cadherin are two important adhesion molecules which are involved in epithelial-mesenchymal transition (EMT), playing a pivotal role in the pathogenesis, development and metastasis of cancer. E-cadherin is regarded as a tumor suppressor factor whereas $\mathrm{N}$-cadherin is related to a poor prognosis in cancer patients (3). The switching between E-cadherin and $\mathrm{N}$-cadherin determines the behavior and characteristics of tumor cells and impacts the progression of cancer and reversing the expression of E-cadherin and $\mathrm{N}$-cadherin is a potential treatment approach. Matrix metalloproteinases (MMPs) are a family of zinc-dependent proteins which have the capacity to degrade ECM and whose function can be inhibited by the tissue inhibitor of metalloproteinases (TIMPs), the role of MMPs and TIMPs in tumor growth, invasion, metastasis and angiogenesis have been investigated in different cancer cells (4). MMP-2 and MMP-9 are enzymes degrade type IV collagen which are strongly expressed in ovarian cancer and are prognostic markers of survival for ovarian cancer patients (5-7).

Tumor spheroids are found in ascites of ovarian cancer patients and exhibit aggressive features which promote adhesion and invasion of cancer cells into peritoneum and omentum, 
resistant to chemotherapy and radiation and are thought to be key contributors to progression of ovarian cancer (8). Ovarian cancer spheroids degrade ECM and facilitate invasion mainly mediated by MMPs, $\beta$-integrin and serine protease (9). A recent study found that ovarian cancer spheroids use $\alpha 5 \beta 1$ integrin to bind to the fibronectin presented by mesothelial cells and mediate displacement of mesothelium allowing attachment and invasion (10). Due to the important role of spheroids in ovarian cancer metastasis, prevention of spheroid formation and growth is an attractive treatment strategy for ovarian cancer.

TSA is a potent histone deacetylase inhibitor with antitumor effect in a mouse model of breast cancer through promotion of histone acetylation which is the basis for anti-neoplastic activity (11). Decitabine, a cytosine analog is used in combination or sequentially with conventional chemotherapeutic agents is able to resensitize chemoresistant ovarian cancer cell lines to platinum or taxane (12). We focused on markers of apoptosis and cell cycle because a recent study reported that decitabine in combination with HDAC inhibitor SAHA (suberoylanilide hydroxamic acid, Vorinostat) suppressed the tumorigenicity of the ovarian cancer cell line SKOV3 and HEY primarily mediated through an increase in apoptosis, additional effects included decreased cellular proliferation, altered cell cycle, increased autophagy, stimulated re-expression of inhibitory imprinted tumor suppressor gene ARHI and PEG3 (13). Demethylating agents and histone deacetylase inhibitors also inhibited proliferation of cells from primary ovarian cancer patient ascites and enhanced cytotoxicity of carboplatin and paclitaxel (14-16).

We hypothesized that the combination of TSA and decitabine has increased anticancer activity on human ovarian ascites cells (SKOV3 cell line) compared to either drug alone. We measured the effect of these drugs on mouse model in vivo, migration and invasion capacity of ovarian cancer cells by transwell and matrigel assays, on spheroid formation, on epigenetic regulation, including the expression of epigenetic associated enzymes DNMTs, HDACs, LSD1 and on the acetylation and methylation of histones.

\section{Materials and methods}

Cell lines and agents. SKOV3 cell line was purchased from American Type Culture Collection (ATCC). Cells were cultured in DMEM/F-12 (1:1) (Gibco) with 10\% FBS (Cellgro) and $1 \%$ penicillin/streptomycin (Gibco). Decitabine (5-aza2'-deoxycytidine, DAC, $10 \mathrm{mg}$ ) and TSA (trichostatin A $5 \mathrm{mM} / 200 \mu \mathrm{l}$ ) were purchased from Sigma-Aldrich. Decitabine was dissolved in DMSO at a concentration of $1 \mu \mathrm{M} / 1$. Spheroid culture medium MammoCult ${ }^{\mathrm{TM}}$ Basic Medium $(450 \mathrm{ml})$ was used for spheroid culture along with MammoCult ${ }^{\mathrm{TM}}$ proliferation supplements $(50 \mathrm{ml})$, hydrocortisone $(1 \mu \mathrm{M} / 1$, $500 \mu \mathrm{l})$, Antibiotic-antimycotic $(5 \mathrm{ml})$ and heparin solution $(0.2 \%, 0.5 \mathrm{ml})$ (StemCell Technology). Transwell ${ }^{\circledR}$ Permeable Supports system (Corning), BD BioCoat ${ }^{\mathrm{TM}}$ BD Matrigel $^{\mathrm{TM}}$ invasion chamber (BD Bioscience), $0.25 \%$ trypsin-EDTA (Gibco), CellTiter ${ }^{\circledR} 96$ Aqueous Non-Radioactive Cell Proliferation assay (Promega) were employed.

Cell viability measurement. Promega Cell Proliferation assay was used for detection of cell viability. The Chou-Talalay median effect and combination index (CI) model was used to determine the effect of combination drug treatments (17). Briefly, SKOV3 cells were treated with each drug individually at multiples $\left(1.0,2.0\right.$ and 3.0) and fraction (0.5) of the $\mathrm{IC}_{50}$ concentration in a fixed ratio (1:50). Combination index was calculated as: $\mathrm{CI}=(\mathrm{D})_{1} /(\mathrm{DX})_{1}+(\mathrm{D})_{2} /(\mathrm{DX})_{2} .(\mathrm{DX})_{1}$ and $(\mathrm{DX})_{2}$ are the concentration of single drugs required to inhibit $\mathrm{x} \%$ of cells and (D) $)_{1}$ and (D) 2 are drugs concentration in combination treatments which also inhibit $\mathrm{x} \%$ of cells. $\mathrm{CI}<1, \mathrm{CI}=1$ and $\mathrm{CI}>1$ indicate synergism, additive and antagonism effect, respectively.

Tumorigenicity of SKOV3 cells. Four- to five-week C3H.CPrkdc/SCID mice were used in this study and the protocol was approved by the Institutional Animal Care and Use Committee of The University of Connecticut Health Center (IACUC). Briefly, SKOV3 cells were pretreated with TSA $0.1 \mu \mathrm{M}$ or the combination of TSA $0.1 \mu \mathrm{M}$ and decitabine $5 \mu \mathrm{M}$ for $48 \mathrm{~h}$ in vitro and then treated cells were incubated in drug-free medium for $4 \mathrm{~h}$ for recovery. Cells were trypsinized and suspended in fresh medium, SKOV3 $\left(1 \times 10^{7}\right)$ cells were injected into the flank of mice subcutaneously and the mice were carefully observed. Tumor size was measured by caliper every 5 days and mice were sacrificed at day 30. Tumor volumes were calculated according to the formula described previously (18).

Cell migration and invasion assay. Transwells were used to measure the migration capacity of cancer cells. In cell migration assay, SKOV3 cells were treated with $0.1 \mu \mathrm{M}$ TSA, $5 \mu \mathrm{M}$ decitabine and TSA plus decitabine for $48 \mathrm{~h}$, then replaced with fresh medium and incubated $4 \mathrm{~h}$ for recovery, then digested with trypsin-EDTA and cell numbers were counted by hemocytometer. SKOV3 cell suspension $(0.5 \mathrm{ml})\left(5 \times 10^{4}\right)$ were seeded into the inserts of transwells and incubated in $37^{\circ} \mathrm{C}$ incubator for $48 \mathrm{~h}$. All the transwell inserts were then washed with fresh 1X PBS, then upper surface of the transwell insert were scraped by cell scraper and washed with fresh $1 \mathrm{X}$ PBS, stained with hematoxylin and eosin and migrated cells in the lower side of the membranes were counted under Olympus IX71 microscope and three fields of triplicate membranes of each group were selected. In cell invasion assay, the protocol of drug treatment was the same as the migration assay, when the cells were harvested and then the cells were seeded into BD Matrigel invasion chamber. After incubation, the noninvading cells were moved from the upper surface of the membrane by cell scraper. The cells on the lower surface of the membrane were stained with hematoxylin and eosin and the number of invaded cells was counted by Olympus IX71 microscope. Three fields of triplicate membranes of each group were selected.

Spheroid formation. Suspended single cells (500 cells $/ \mathrm{ml}$ ) were mixed with combined drugs and seeded in 24-well ultralow attachment plates (Corning) in spheroid culture medium and incubated at $37^{\circ} \mathrm{C}$ for 15 days and images were recorded every 5 days. To further investigate the spheroid formation ability (spheroid number), $1 \times 10^{3}$ suspended cells were seeded simultaneously with drugs in 96-well ultra-low attachment plates (Corning) and incubated at $37^{\circ} \mathrm{C}$ for 15 days. The 
number of spheroids were counted and the difference between untreated and combined treated group was compared using Student's t-test.

In vivo implantation assay. SKOV3 $\left(1 \times 10^{7}\right)$ cells were mixed with TSA $(0.1 \mathrm{mg} / \mathrm{kg})$ and decitabine $(5 \mathrm{mg} / \mathrm{kg})$ and then injected into 4-5-week C3H.C-Prkdc/SCID mice intraperitoneally for total treatment of 5 days. Mice were sacrificed on day 30 from the first day of inoculation. The number and volume of implanted xenograft nodules were assessed and expression of apoptosis related proteins Bcl-2, Bcl-xL, p21, p27 of tumors were detected by western blotting.

Western blotting. Pretreated SKOV3 cells or cancer cells from implanted xenograft nodules were digested and total protein was quantified by Bradford Protein assay (Bio-Rad), protein was denatured in 2X laminin sample buffer (Sigma-Aldrich), 10-30 $\mu \mathrm{g} /$ lane samples were loaded and separated on $7-10 \%$ SDS-PAGE gel, the gels were transferred to nitrocellulose membrane (Whatman), membranes were blocked in 5\% non-fat milk (Lab Scientific) for $1 \mathrm{~h}$ and then primary antibodies were added and incubated at $4^{\circ} \mathrm{C}$ overnight. The antibodies and dilution were: E-cadherin, N-cadherin, Twist, DNMT3A, DNMT3B, HDAC1, HDAC2, LSD1 (1:1000, Cell Signaling Technology), Bcl-2, Bcl-xL, p21, p27, MMP-2, MMP-9 (1:500 Santa Cruz Biotechnology) and $\beta$-actin (1:10,000 SigmaAldrich). Then, the membranes were washed with fresh 1X TBST solution three times for $10 \mathrm{~min}$ and incubated with secondary antibodies (Santa Cruz Biotechnology) for $1 \mathrm{~h}$ at room temperature, after being washed with fresh 1X TBST solution three times for $10 \mathrm{~min}$. SuperSignal ${ }^{\circledR}$ West Femto Maximum chemiluminescent substrate (Thermo Scientific) was added to membranes and detected by luminescent detection system (Syngene system) and densitometric values of western blotting were assessed by ImageJ software (NIH. gov).

Histone immunoblots. Histone protein of treated SKOV3 cells was extracted and purified by the Epiquick total histone extraction kit (Epigenetek), the extraction was quantified and then diluted in 1X NuPAGE/LDS loading buffer (Invitrogen), heated at $95^{\circ} \mathrm{C}$ in a dry heater for $5 \mathrm{~min}, 10-30 \mu \mathrm{g} /$ lane samples were loaded in 10\% NuPAGE/Bus/Tris gel (Invitrogen), run in NuPAGE/MES/SDS buffer (Invitrogen), transferred into nitrocellulose membranes, blocked in 5\% BSA/TBST solution for $1 \mathrm{~h}$ and incubated with primary antibodies at $4^{\circ} \mathrm{C}$ overnight. Membranes were washed by $1 \mathrm{X}$ TBST solution and incubated with secondary antibodies for $1 \mathrm{~h}$ at room temperature. The subsequent protocol was the same as described in the western blotting. The antibodies and dilution were: H3K4me2, H3K9me2 and histone H3 (1:1,000, Cell Signaling Technology).

Statistical analysis. SPSS 16.0 (IMB, Armonk, NY) was used to analysis the data, GraphPad Prism 5 (GraphPad Software, San Diego, CA) was used for making graphs and ImageJ software (NIH.gov) was used to assess the densitometric readings of western blotting, the comparison of more than two groups was evaluated by one-way ANOVA and the difference between two groups was evaluated by independent Student's t-test, $\mathrm{P}<0.05$ was considered statistically significant.

\section{Results}

Effect of TSA and decitabine on tumorigenicity of SKOV3 cells in a mouse xenograft model. DNA demethylation agents and histone deacetylase inhibitor reported to suppress tumor formation when used alone or combined with conventional cytotoxic drugs in cancers (12). We first assessed the effect of fixed ratio combination of TSA and decitabine on the cell viability and the results indicated that the combination of TSA $0.1 \mu \mathrm{M}$ and decitabine $5 \mu \mathrm{M}$ achieved the ultimate synergistic effect $(\mathrm{CI}=0.421)$ and this combination was selected for subsequent experiments (Fig. 1A). To determine if the combination of TSA and decitabine was better than either drug alone in suppressing xenograft tumor formation with SKOV3 cells, we evaluated the tumorigenicity of pretreated SKOV3 cells in mouse xenograft models. We found that the combination suppressed tumor formation greater than either agent used alone (Fig. 1B). There was a statistically significant difference between tumor volume and weight from the cells pretreated with single drugs compared to the combination (Fig. 1C-E), suggesting a profound effect on tumorigenesis only by pretreating cells with the combination of agents.

Effect of TSA and decitabine on the migration and invasion of SKOV3 cells in vitro. Migration and invasion play an important role in the pathogenesis, development and metastasis of ovarian cancer (2). Migration capacity was measured in SKOV3 cells which were treated with TSA alone or the combination of TSA and decitabine. TSA/decitabine suppressed migration more than either drug alone (Fig. 2A and B) and induced reversal of epithelial to mesenchymal transition (EMT) promoting a switch from $\mathrm{N}$-cadherin to E-cadherin (Fig. 2D). We also found that invasion capacity of SKOV3 cells was almost totally suppressed by the combination of TSA and decitabine (Fig. 2C), the expression levels of MMP-2 and MMP-9 were significantly suppressed by the combined treatment (Fig. 2D). The upregulation of E-cadherin, downregulation of N-cadherin and suppression of MMP-2 and MMP-9 may suppress metastasis by inhibiting migration and ECM degradation.

The combination of TSA and decitabine suppresses spheroid formation via regulation of EMT in vitro. Spheroids, normally occurring in ovarian cancer patients, show enhanced migration, invasion and motility and are thought to play an important role in the metastasis of ovarian cancer (19). When grown in serumfree medium, SKOV3 cells formed round, tightly adhered spheroids which were inhibited with the combination of drugs (Fig. 3A and B). Migration and invasion ability of spheroid derived cells were then assessed and both of these functions were impaired when compared with untreated group (Fig. 3C and D). The expression of several EMT markers was detected and the results indicated that Twist and $\mathrm{N}$-cadherin were significantly suppressed, E-cadherin was markedly induced and both MMP-2 and MMP-9 were markedly suppressed by the combined drugs (Fig. 3E).

The combination of TSA and decitabine suppresses tumor metastases and implantation through apoptosis pathway in vivo. Based on the spheroid assay results, in order to further assess if the combination of TSA and decitabine could have 
A

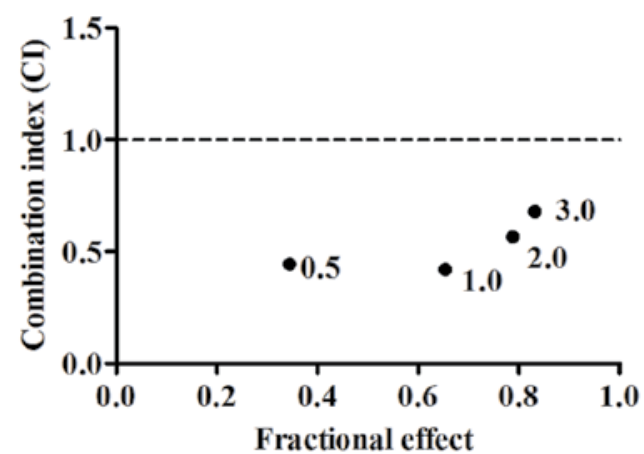

C

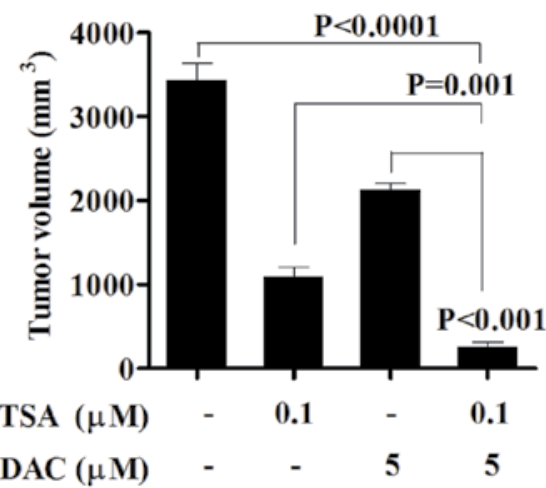

B

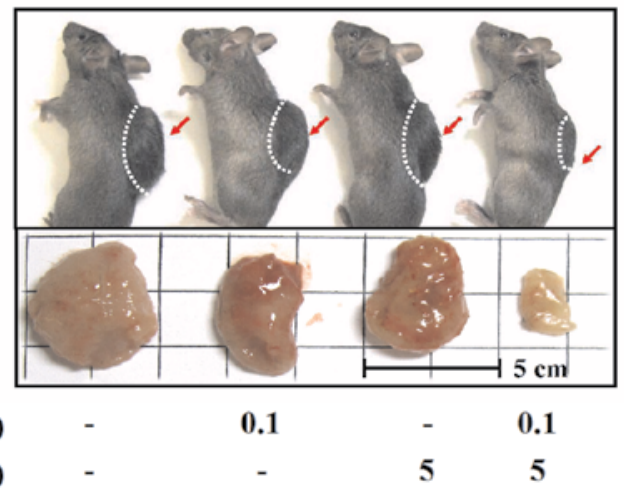

E
D

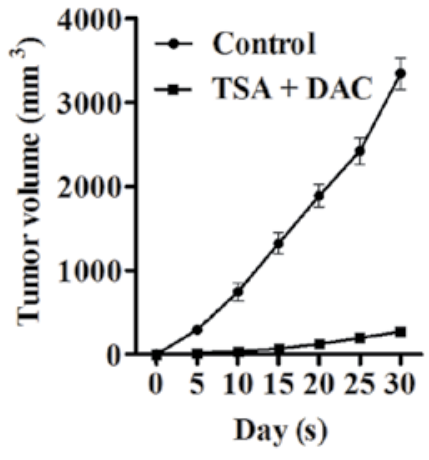

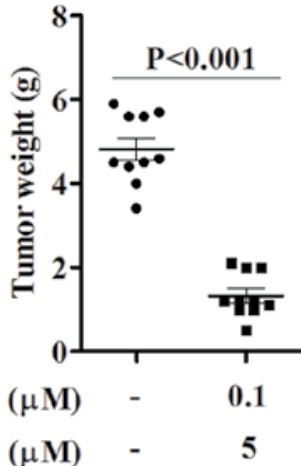

Figure 1. The combination of TSA and decitabine significantly suppressed the tumorigenicity of SKOV3 in a mouse model. (A) SKOV3 cells were exposed to a fixed ratio doses of TSA and decitabine and the cell viability was detected by MTS assay. The number adjacent to the spot $(0.5,1.0,2.0$ and 3.0$)$ indicates fraction and multiples of the $\mathrm{IC}_{50}$ of TSA $\left(\mathrm{IC}_{50}=0.1 \mu \mathrm{M}\right)$ and decitabine $\left(\mathrm{IC}_{50}=5 \mu \mathrm{M}\right)$. CI $<1$ indicates synergistic effect. (B) Representative images of tumors from untreated group and treatment groups. (C and D) Tumor volume and growth curve of SKOV3 xenograft tumor. The results are presented as mean $\pm S E M, n=10, P<0.05$ was considered statistically significant; control vs. treatment group or single treatment vs. combination. (E) Tumor weights were recorded and assessed.

A

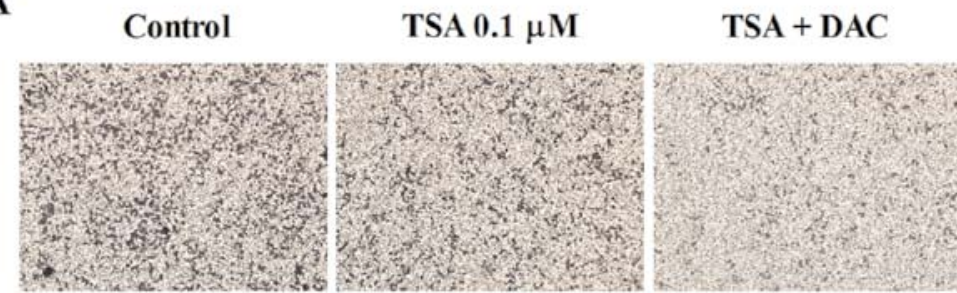

B

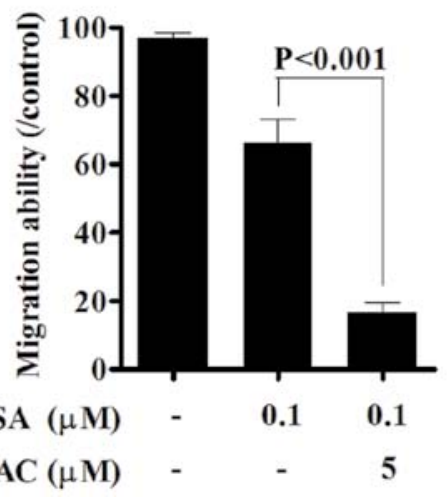

C

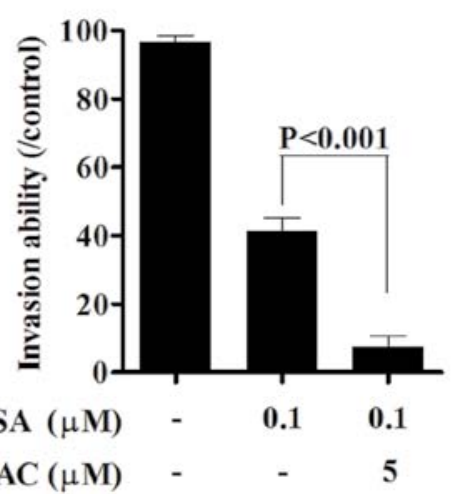

D

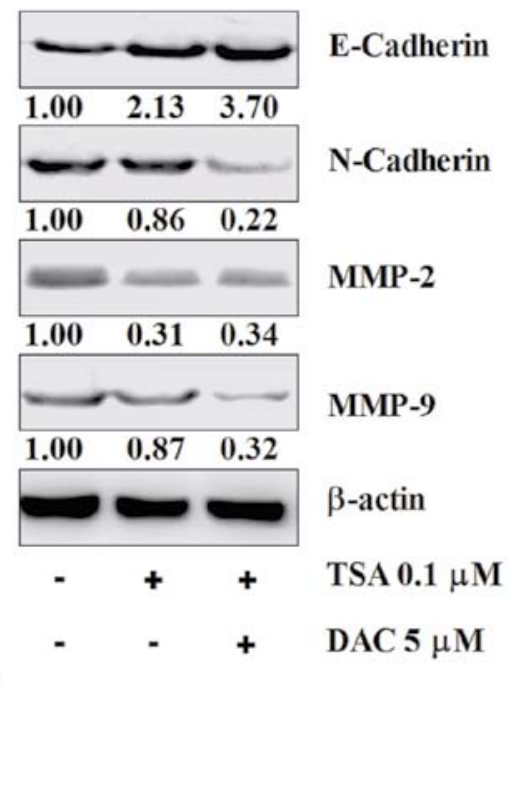

Figure 2. Migration and invasion of SKOV3 cells were significantly suppressed by the combination of TSA and decitabine via regulation of cadherins and MMPs. (A and B) SKOV3 cells were exposed to single or combined drugs and the migration capacity was detected with transwell assay. TSA+DAC, trichostatin A $0.1 \mu \mathrm{M}$ plus decitabine $5 \mu \mathrm{M}$. (C) SKOV3 cells treated with TSA alone or the combination of TSA and decitabine in vitro and invasion capacity of SKOV3 cells was assessed with BD Matrigel invasion assay. (D) Expression levels of E-cadherin, N-cadherin, MMP-2 and MMP-9 were measured by western blotting, the numbers beneath the blots are the densitometry readings of the protein quantity relative to control. $\beta$-actin is a loading control. 
A

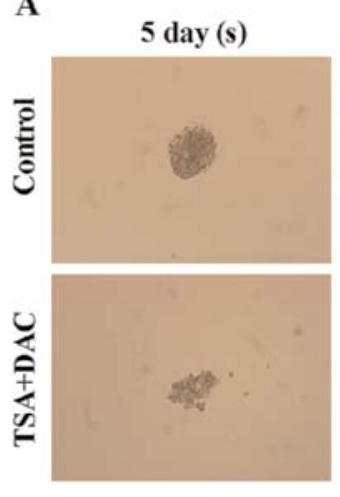

B

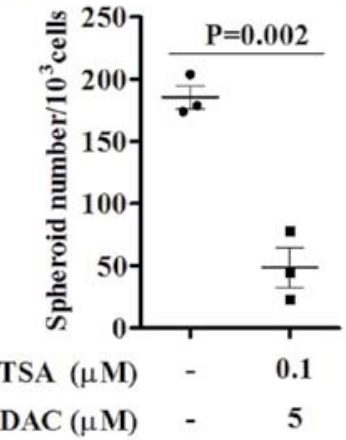

10 day $(s)$

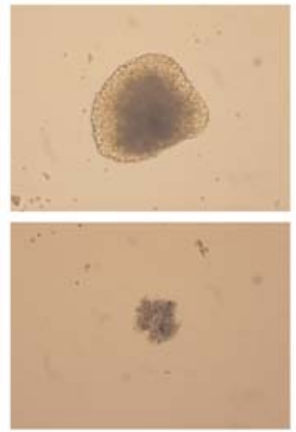

C
15 day $(\mathbf{s})$

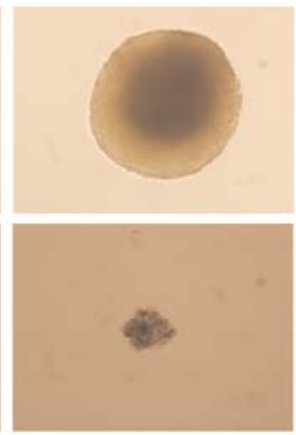

D
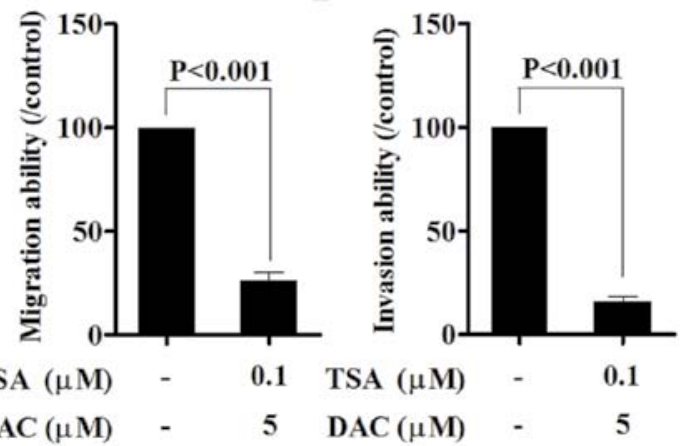

$\mathbf{E}$

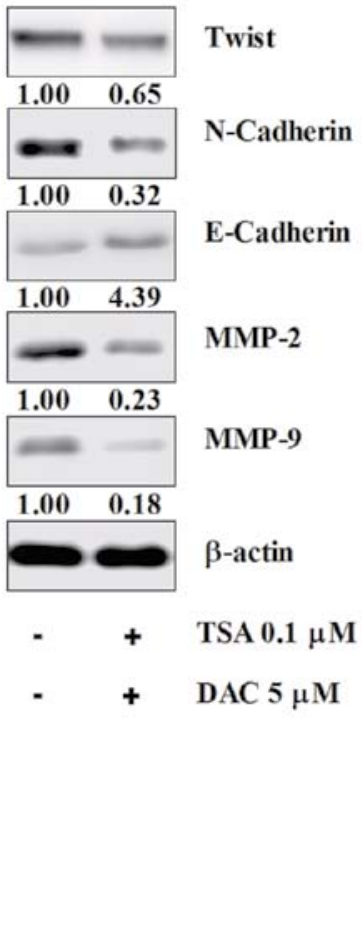

Figure 3. Effect of the combination of TSA and decitabine on spheroid formation in vitro. (A) Suspended SKOV3 cells were seeded with or without the combination drugs in ultra-low attachment plates in vitro. TSA+DAC, trichostatin A $0.1 \mu \mathrm{M}$ plus decitabine $5 \mu \mathrm{M}$. (B) Spheroid number was counted when the cells and drugs were co-cultured in 96-wells for 15 days. Data are presented as the mean \pm SEM of three independent experiments. (C and D) The migration and invasion ability of cells which were derived from spheroids were assessed. (E) The expression levels of EMT markers were detected.

A

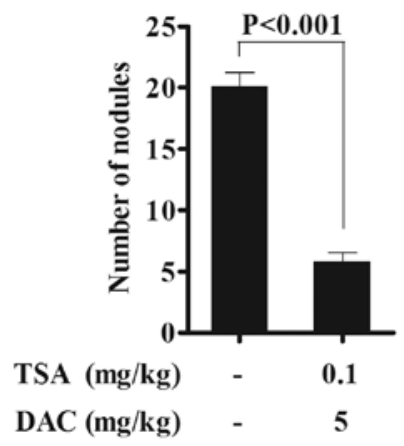

B

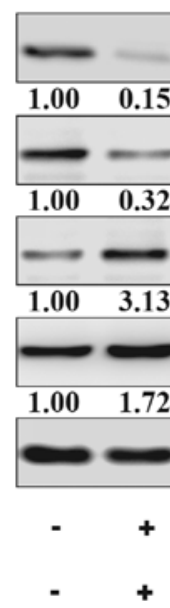

Bcl-2

Bcl-xL

p21

p27

$\beta$-actin

TSA $0.1 \mathrm{mg} / \mathrm{kg}$

DAC $5 \mathrm{mg} / \mathrm{kg}$

Figure 4. Effect of the combination of TSA and decitabine on tumor xenograft implantation in vivo. (A) The numbers of IP nodules of drug treated mice were counted and compared to that of untreated group. Data are presented as the mean \pm SEM, $n=10$. (B) The expression of apoptosis related proteins of implanted nodules were detected. (C) Body weights of mice were measured and compared (treatment group vs. control, Student's t-test).

anticancer effect in abdominal cavity in mouse models, we performed in vivo treatment assay with the combined drugs. There was a significant reduction in peritoneal cavity cancer cell implants when TSA and decitabine were administered intraperitoneally for 5 days following IP injection of SKOV3 cells (Fig. 4A). Bcl-2 and Bcl-xL were significantly suppressed and p21 and p27 were slightly induced in implanted tumor nodules in animals treated with the combination drugs (Fig. 4B). Body weight of untreated and treated mice were measured and there was no significant weight loss or adverse side effects although body weight difference was $\mathrm{P}=0.0537$ (Fig. 4C). The animal results suggested that continuous combined treatment suppress tumor implantation through inhibiting spheroid formation by activation of the apoptosis pathway without any apparent drug toxicity.

Effect of TSA and decitabine on the expression of DNMTs/ HDACs/LSDI and the expression of acetylation of histone H3, 
A

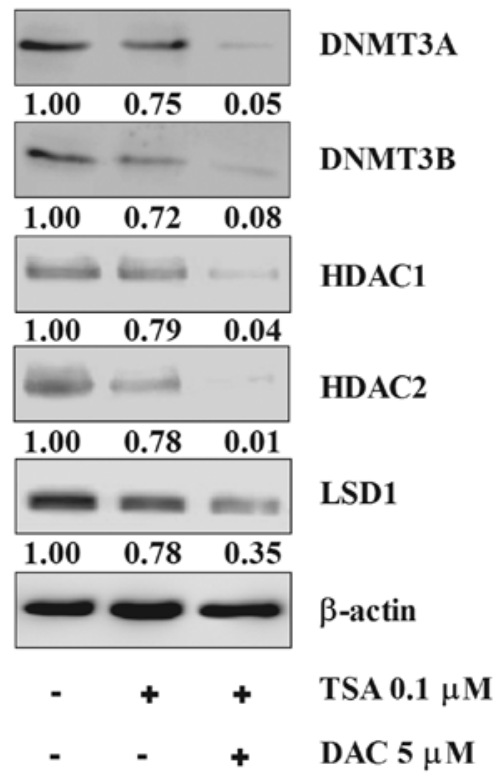

B

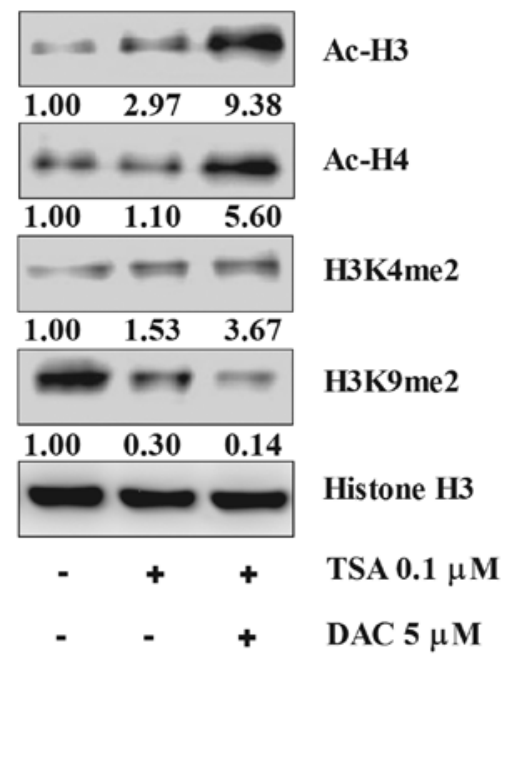

Figure 5. Epigenetic regulation of ovarian cancer cells by the combination of TSA and decitabine. (A) SKOV3 cells were exposed to TSA $0.1 \mu$ M or the combination and the expression of DNMT3A/3B, HDAC1/2 and LSD1 were detected. $\beta$-actin is a loading control. (B) Expression of acetylation of histone H3 and $\mathrm{H} 4$ and histone methylation markers $\mathrm{H} 3 \mathrm{~K} 4 \mathrm{me} 2$ andH3K9me2 were assessed. Histone $\mathrm{H} 3$ is a loading control.

- $H 4$ and $H 3 K 4 m e 2, H 3 K 9 m e 2$. Essential epigenetic associated enzymes DNMT3A/3B, HDAC1/2 and LSD1 were significantly suppressed by the combination whereas TSA alone moderately decreased expression (Fig. 5A). Acetylation status of histone $\mathrm{H} 3$ and $\mathrm{H} 4$, were significantly stimulated and $\mathrm{H} 3 \mathrm{~K} 4 \mathrm{me} 2$ was significantly induced but $\mathrm{H} 3 \mathrm{~K} 9 \mathrm{me} 2$ was suppressed by TSA alone and the combination, with the combination having more effect than TSA alone (Fig. 5B). These results suggest that tumor suppression effect of SKOV3 xenografts by the combination of TSA and decitabine are at least partially epigenetically regulated and the combination of multi-target epigenetic inhibitors achieves a synergistic effect.

\section{Discussion}

In order to metastasize, ovarian cancer cells must have the ability to detach from their primary location, invade and migrate into ECM, reattach to the omentum and peritoneum, and these processes are mainly mediated by downregulation of cell-cell adhesion and upregulation of ECM degradation.

The expression of E-cadherin have been shown to be regulated at multiple levels, transcriptionally, translationally and post-translationally. Several important epigenetic associated regulators have been found to be involved in the regulation of E-cadherin both at the epigenetic and transcription levels. A recent study reported that the presence of LDS1 is a prerequisite for the repression of E-cadherin by Snail through demethylation of dimethylated H3K4 and suggested that BRAF-HDAC complex could be a potential target for the prevention of EMT-associated tumor invasion (20). Snail mediated the expression of E-cadherin by interacting with HDACs and the addition of TSA is sufficient to block the repressive effect of Snail on E-cadherin (21). Twist is a transcriptional factor, which is able to promote metastasis of epithelial cancer cells through regulating the process of EMT, inhibiting the expression of E-cadherin by binding to E-cadherin promoter and activating expression of the mesenchymal marker $\mathrm{N}$-cadherin $(22,23)$. E-cadherin is epigenetically regulated and upregulation of E-cadherin suppressed migration and invasion. E-cadherin and $\gamma$-catenin could be markedly induced by the simultaneous inhibition of DNMT3A and DNMT3B with the addition of decitabine in choriocarcinoma cells (24). Our findings are consistent with these data and suggest that the inhibition of migration and invasion of ovarian cancer cells with the combination of TSA and decitabine is mediated through inhibition of HDACs and LSD1, which in turn, blocks the suppression of E-cadherin and activation of Twist and N-cadherin.

The role of MMPs is far more than cleavage of basement membrane to facilitate invasion into the omentum and the peritoneal cavity in ovarian cancer. Studies have found that MMP-9 triggers angiogenesis and the stromal MMP-9 promotes blood vessel formation and pericyte recruitment to angiogenesis in neuroblastoma (25). Recent reports demonstrated that downregulation of MMP-2 mRNA or inhibition of the proteolytic activity of MMP-2 in both human ovarian primary cells and cell lines reduced the attachment of ovarian cancer cells to the peritoneum and omentum in vitro and in vivo. The cleavage of vitronectin and fibronectin, which is highly expressed in the mesothelial cells of the lining of peritoneal cavity, is mediated by MMP-2 which is considered to be the iniating event in ovarian cancer metastasis $(6,7)$. Inhibiting the secretion and proteolytic activity of MMP-2 would be vital to the prevention of cancer cell metastasis and colonization and our data show that the combination of TSA and decitabine significantly inhibited the expression of MMP-2 and MMP-9 and inhibited ovarian cancer invasion. If ovarian cancer in women could be induced to undergo the reverse of EMT with upregulation of E-cadherin and downregulation of $\mathrm{N}$-cadherin as well as 
modulation of the MMPs necessary for invasion, this could be a major breakthrough to prevent recurrence of this cancer.

Epigenetic alterations, including DNA methylation and histone modification, contribute to ovarian cancer progression and drug resistance (26). Intimate crosstalk exists between histone modification and DNA methylation. DNA demethylating agents and HDAC inhibitors are two classes of epigenetic modifiers which have been extensively studied and show promising anticancer efficacy in chemoresistant ovarian cancers (27). DNMTs are thought to be important in the pathogenesis and progression of ovarian cancers. The expression of DNMTs is suppressed by the histone deacetylase inhibitor TSA, with the effect of HDAC inhibitor on DNMTs mainly associated with the spatiotemporal regulation of DNA methylation and histone modification (28). Our data suggest that the combination of a DNMT inhibitor and HDAC inhibitor could achieve significant epigenetic regulation effect via the stimulation of acetylation of histone $\mathrm{H} 3 / 4$ and suppression of epigenetic related enzymes, such as HDACs and DNMTs.

Histone methylation is involved in gene transcription, whether it functions to activate or repress mainly depends on the number of the methyl groups. Two of the best studied methylation markers are transcription activation markers H3K4me2 and repression markers H3K9me2 (29). The demethylated-H3K4 could be demethylated by LSD1 and demethylated-H3K9 can also be demethylated by LSD1 in presence of the androgen receptor (30). Our study demonstrated the LSD1 was inhibited by TSA and decitabine and suggested that interactive regulation exists among DNA methylation, histone deacetylation and histone methylation. Moreover, our previous data showed that TSA or decitabine in combination of cisplatin could significantly suppress the expression of LSD1 and HDAC1/2 in SKOV3 cells (data not published). We thus hypothesize that combining epigenetic modifiers potentiate their anticancer effect.

Based on our results, suppression of tumorigenicity in vivo and the inhibitory effect on migration and invasion in vitro, we hypothesized that the combination of TSA and decitabine could also affect the implantation of cancer cells in vivo. The suppression of spheroid formation and viability could significantly impact the ability of ovarian cancer to recur with metastasis and invasion. The major application of this approach may lie in prolonging remission in women in clinical remission following chemotherapy or treating recurrent disease.

\section{Acknowledgements}

This study was supported by The Carole and Ray Neag Comprehensive Cancer Center, University of Connecticut Health Center.

\section{References}

1. Bast RC Jr, Hennessy B and Mills GB: The biology of ovarian cancer: new opportunities for translation. Nat Rev Cancer 9: 415-428, 2009

2. Lengyel E: Ovarian cancer development and metastasis. Am J Pathol 177: 1053-1064, 2010.

3. Ahmed N, Thompson EW and Quinn MA: Epithelialmesenchymal interconversions in normal ovarian surface epithelium and ovarian carcinomas: an exception to the norm. J Cell Physiol 13: 581-588, 2007.
4. Roy R, Yang J and Moses MA: Matrix metalloproteinases as novel biomarkers and potential therapeutic targets in human cancer. J Clin Oncol 27: 5287-5297, 2009.

5. Sillanpää S, Anttila M, Voutilainen K, Ropponen K, Turpeenniemi-Hujanen T, Puistola U, Tammi R, Tammi M, Sironen R, Saarikoski S and Kosma VM: Prognostic significance of matrix metalloproteinase-9 (MMP-9) in epithelial ovarian cancer. Gynecol Oncol 104: 296-303, 2007.

6. Kenny HA, Kaur S, Coussens LM and Lengyel E: The initial steps of ovarian cancer cell metastasis are mediated by MMP-2 cleavage of vitronectin and fibronectin. J Clin Invest 118: 1367-1379, 2008

7. Kenny HA and Lengyel E: MMP-2 functions as an early response protein in ovarian cancer metastasis. Cell Cycle 8: 683-688, 2009

8. Burleson KM, Casey RC, Skubitz KM, Pambuccian SE, Oegema TR Jr and Skubitz AP: Ovarian carcinoma ascites spheroids adhere to extracellular matrix components and mesothelial cell monolayers. Gynecol Oncol 93: 170-181, 2004

9. Burleson KM, Hansen LK and Skubitz AP: Ovarian carcinoma spheroids disaggregate on type I collagen and invade live human mesothelial cell monolayers. Clin Exp Metastasis 21: 685-697, 2004.

10. Iwanicki MP, Davidowitz RA, Ng MR, Besser A, Muranen T, Merritt M, Danuser G, Ince TA and Brugge JS: Ovarian cancer spheroids use myosin-generated force to clear the mesothelium. Cancer Discov 1: 144-157, 2011.

11. Vigushin DM, Ali S, Pace PE, Mirsaidi N, Ito K, Adcock I and Coombes RC: Trichostatin A is a histone deacetylase inhibitor with potent antitumor activity against breast cancer in vivo. Clin Cancer Res 7: 971-976, 2001.

12. Kristensen LS, Nielsen HM and Hansen LL: Epigenetics and cancer treatment. Eur J Pharmacol 625: 131-142, 2009.

13. Chen MY, Liao WS, Lu Z, Bornmann WG, Hennessey V, Washington MN, Rosner GL, Yu Y, Ahmed AA and Bast RC Jr: Decitabine and suberoylanilide hydroxamic acid (SAHA) inhibit growth of ovarian cancer cell lines and xenografts while inducing expression of imprinted tumor suppressor genes, apoptosis, G2/M arrest and autophagy. Cancer 117: 4424-4438, 2011.

14. Li Y, Hu W, Shen DY, Kavanagh JJ and Fu S: Azacitidine enhances sensitivity of platinum-resistant ovarian cancer cells to carboplatin through induction of apoptosis. Am J Obstet Gynecol 200: 177 e1-e9, 2009.

15. Sonnemann J, Gänge J, Pilz S, Stötzer C, Ohlinger R, Belau A Lorenz $\mathrm{G}$ and Beck JF: Comparative evaluation of the treatment efficacy of suberoylanilide hydroxamic acid (SAHA) and paclitaxel in ovarian cancer cell lines and primary ovarian cancer cells from patients. BMC Cancer 6: 183, 2006.

16. Dietrich CS III, Greenberg VL, DeSimone CP, Modesitt SC, van Nagell JR, Craven R and Zimmer SG: Suberoylanilide hydroxamic acid (SAHA) potentiates paclitaxel induced apoptosis in ovarian cancer cell lines. Gynecol Oncol 116: 126-130, 2010.

17. Chou TC: Drug combination studies and their synergy quantification using the Chou-Talalay method. Cancer Res 70: 440-446, 2010.

18. Baba T, Convery PA, Matsumura N, Whitaker RS, Kondoh E, Perry T, Huang Z, Bentley RC, Mori S, Fujii S, Marks JR, Berchuck A and Murphy SK: Epigenetic regulation of CD133 and tumorigenicity of $\mathrm{CD} 133^{+}$ovarian cancer cells. Oncogene 28: 209-218, 2009.

19. Shield K, Ackland ML, Ahmed N and Rice GE: Multicellular spheroids in ovarian cancer metastases: biology and pathology. Gynecol Oncol 113: 143-148, 2009.

20. Lin T, Ponn A, Hu X, Law BK and Lu J: Requirement of the histone demethylase LSD1 in Snai1-mediated transcriptional repression during epithelial-mesenchymal transition. Oncogene 29: 4896-4904, 2010.

21. Peinado H, Ballestar E, Esteller M and Cano A: Snail mediates E-cadherin repression by the recruitment of the Sin3A/histone deacetylase 1 (HDAC1)/HDAC2 complex. Mol Cell Biol 24: 306-319, 2004.

22. Yang J, Mani SA, Donaher JL, Ramaswamy S, Itzykson RA, Come C, Savagner P, Gitelman I, Richardson A and Weinberg RA: Twist, a master regulator of morphogenesis, plays an essential role in tumor metastasis. Cell 117: 927-939, 2004.

23. Vesuna F, van Diest P, Chen JH and Raman V: Twist is a transcriptional repressor of E-cadherin gene expression in breast cancer. Biochem Biophys Res Commun 367: 235-241, 2008. 
24. Rahnama F, Shafiei F, Gluckman PD, Mitchell MD and Lobie PE: Epigenetic regulation of human trophoblastic cell migration and invasion. Endocrinology 147: 5275-5283, 2006.

25. Bergers G, Brekken R, McMahon G, Vu TH, Itoh T, Tamaki K, Tanzawa K, Thorpe P, Itohara S, Werb Z and Hanahan D: Matrix metalloproteinase-9 triggers the angiogenic switch during carcinogenesis. Nat Cell Biol 2: 737-744, 2000.

26. Chen H, Hardy TM and Tollefsbol TO: Epigenomics of ovarian cancer and its chemoprevention. Front Genet 2: 67, 2011.

27. Balch C, Huang TH, Brown R and Nephew KP: The epigenetics of ovarian cancer drug resistance and resensitization. Am J Obstet Gynecol 191: 1552-1572, 2004.
28. Balch C, Fang F, Matei DE, Huang TH and Nephew KP: Minireview: epigenetic changes in ovarian cancer. Endocrinology 150: 4003-4011, 2009.

29. Yoo CB and Jones PA: Epigenetic therapy of cancer: past, present and future. Nat Rev Drug Discov 5: 37-50, 2006.

30. Metzger E, Wissmann M, Yin N, Müller JM, Schneider R, Peters AH, Günther T, Buettner R and Schüle R: LSD1 demethylates repressive histone marks to promote androgen-receptor dependent transcription. Nature 437: 436-439, 2005. 